\title{
Long-term follow-up evaluation of chronic pain after endoscopic total extraperitoneal repair of primary and recurrent inguinal hernia
}

\author{
A. E. M. van der Pool • J. J. Harlaar • \\ P. T. den Hoed $\cdot$ W. F. Weidema $\cdot$ R. N. van Veen
}

Received: 6 August 2009/ Accepted: 5 December 2009/Published online: 7 January 2010

(C) The Author(s) 2010. This article is published with open access at Springerlink.com

\begin{abstract}
Background Long-term data on chronic pain after endoscopic total extraperitoneal (TEP) hernia repair are hardly available.

Methods Between January 1997 and December 1998, 416 patients with consecutive primary and recurrent inguinal hernia underwent endoscopic TEP hernia repair. Long-term follow-up evaluation was carried out from June 2007 to June 2008. The primary outcome measure was persistent pain and discomfort interfering with daily activity.

Results The overall response rate was 66\% (273 of 416 patients). Of the 416 patients, 85 (20\%) had died of causes unrelated to hernia repair and 58 (14\%) were lost to followup. A total of 177 patients were physically examined in the outpatient clinic. Because 96 patients were not able to visit the outpatient' clinic, they completed the survey by telephone. The median follow-up period was 10 years (range, 9-11 years). After TEP repair, 16 patients (6\%) reported chronic groin pain, and 10 patients (4\%) still experience pain at this writing after the 10-year follow-up period. One of the patients has experienced persistent pain and discomfort interfering with daily activity. Patients with preoperative pain have reported significantly more chronic pain $(P=0.03)$.
\end{abstract}

\footnotetext{
A. E. M. van der Pool · P. T. den Hoed .

W. F. Weidema · R. N. van Veen $(\bowtie)$

Division of Surgery, Ikazia Hospital, Montessoriweg 1,

3083 AN Rotterdam, The Netherlands

e-mail: r.n.vanveen@erasmusmc.nl

J. J. Harlaar

Division of Surgery, Erasmus University MC, Rotterdam,

The Netherlands
}

Conclusions Chronic groin pain after TEP repair of primary and recurrent inguinal hernia seems to have a low incidence after a 10-year follow-up period.

Keywords Chronic pain - Endoscopic - Inguinal hernia · Long-term follow-up · TEP

The use of prosthetic mesh allows tension-free inguinal hernia repair. Concomitant with popularization of this repair, hernia recurrence is a relatively small problem, occurring after $1-10.1 \%$ of mesh repairs. Similar outcomes after open and endoscopic hernia repair have been reported [1-5]. Because recurrence rates have been reduced, hernia repair research has recently focused on chronic pain [6].

Chronic groin pain represents the most common postoperative morbidity after open repair of inguinal hernia. Several studies have reported a high prevalence of chronic pain $[7,8]$. A recent review reported chronic pain experienced by $11 \%$ of patients undergoing mesh-based inguinal hernia repair, with one-third reporting limitation in daily leisure activities [9]. The most common types of chronic postoperative pain are somatic and neuropathic $[8,10]$.

Because many of the operations that produce persistent pain are associated with risk of damage to major nerves, techniques to avoid such damage merit investigation [10, 11]. Despite the severity of pain experienced by individual patients in the short term, chronic postoperative pain of neuropathic or somatic origin after open anterior mesh repair seems to dissipate over time [12].

Endoscopic repairs are known to be less painful than open repairs in the early period after surgery [13]. In 2007, a metaanalysis with a mean follow-up period of 20.5 months (range, 3-82 months) favored the endoscopic 
approach to open anterior mesh repair for chronic pain after inguinal hernia repair [14]. Long-term data on chronic pain after endoscopic totally extraperitoneal (TEP) hernia repair are hardly available. Follow-up data on chronic pain after 10 years need to be assessed to determine whether endoscopic repair is favorable in the long term. This study aimed to provide these long-term results of chronic groin pain after TEP repair.

\section{Materials and methods}

Between January 1997 and December 1998, 416 consecutive patients underwent an elective endoscopic TEP inguinal hernia repair in the Ikazia Hospital at Rotterdam, The Netherlands. A polypropylene prosthetic mesh placement $(10 \times 15 \mathrm{~cm})$ was used, as described previously [5, 15]. The prosthesis was anchored to the abdominal wall by intraabdominal pressure, and no fixation materials were used. All the procedures were performed with the patient under general anesthesia, and data on patient characteristics, hernia, and procedure were collected. All operations were performed under the supervision of three staff surgeons highly experienced in endoscopic surgery. These surgeons had performed more than 100 TEP repairs in 4 years (1993-1996).

Postoperative analgesia was prescribed at the discretion of the operating surgeon or the patient's general practitioner after discharge from the hospital. Follow-up evaluation with physical examination was performed at the outpatient clinic within 2 weeks. Postoperative pain was defined as groin pain or discomfort within 3 months after the operation. Chronic pain was defined as continuous pain more than 3 months after inguinal hernia repair, the period regarded as the normal tissue-healing time.

Long-term follow-up evaluation occurred from June 2007 to June 2008. All medical records were reviewed in January 2009. Patients were invited to visit the outpatient clinic. If unable to visit the outpatient clinic, they were asked to participate in a survey by telephone. Physical examination was performed by one of the authors who did not perform the initial hernia repair.

The participants were asked to rate their postoperative and chronic pain on a 10-point visual analog scale (VAS). A score lower than 1 was graded as mild pain, a score of 15 as moderate pain, and a score exceeding 5 as severe pain [7]. The groin region was examined physically for recurrence of inguinal hernia, defined as a symptomatic or asymptomatic defect (bulge or weakness) in the abdominal wall of the operative area with herniation of abdominal contents outside the external ring exacerbated by Valsalva maneuver. Ultrasound examination was performed when physical examination was not conclusive.
Patients who underwent the survey by telephone were asked to rate postoperative pain and chronic pain on a 10-point numeric rating scale. They were quizzed about the severity of the pain, its nature and frequency, and its impact on daily activities. The patients were asked whether they had undergone hernia repair since their last visit.

Because no validated and internationally accepted method exists to distinguish nociceptic, neuropathic, and visceral pain after inguinal hernia repair without quantitative sensory testing, we differentiated between continuous and discontinuous pain $[6,16,17]$. Discontinuous pain existed if pain was reported after certain movements or after repetitive exercises. The maximal pain score during these pain episodes was analyzed in this study.

Categorical data are presented as percentage frequencies. Differences between proportions were compared using the chi-square test or Fisher's exact test, as appropriate. The mean values of continuous variables with normal distributions were compared using the unpaired Student's $t$-test. Continuous data with a significantly skewed distribution are expressed as medians and were compared using the Mann-Whitney $U$-test. A $P$ value of 0.05 (two-sided) was considered the limit of significance. All statistical analyses were performed using the Statistical Package for Social Sciences for Windows version 15.0 (SPSS Inc., Chicago, IL, USA).

\section{Results}

The overall response rate was $66 \%$ (273 of 416 patients). Of the 416 patients, 85 (20\%) had died of causes unrelated to hernia repair, and $58(14 \%)$ were lost to follow-up evaluation. A total of 177 patients visited the outpatient clinic. Because 96 patients were unable to visit the outpatient' clinic, they completed the survey by telephone (Fig. 1). The mean age of the total study group $(n=273)$ was $54 \pm 14$ years, and most of the participants were men (94\%). Inguinal hernias were unilateral for 233 patients (85\%) (125 right, 108 left) and bilateral for 40 patients $(15 \%)$. A total of 33 patients (12\%) underwent TEP repair for recurrent hernia. The median interval between surgery and long-term follow-up period was 10 years (range, 911 years).

\section{Chronic pain}

Chronic groin pain ( $>3$ months continuous pain after resection) was reported by 17 patients (6\%). For 15 patients, pain severity did not change over the study period. One patient reported slightly progressive pain over the specified period. One patient had no postoperative pain but experienced mild chronic pain (VAS 2) the final 3 years. 


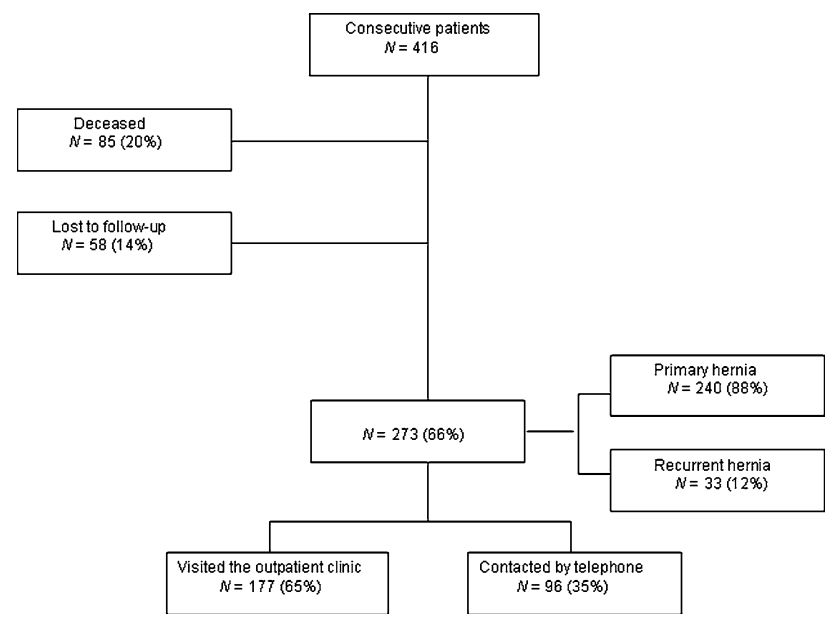

Fig. 1 Flow chart

At this writing, 10 patients still experience chronic pain after a 10-year follow-up period. One patient with chronic groin pain (patient 9, Fig. 2) was consulted 1 year after hernia repair with a recurrence. He was advised to lose weight before undergoing additional surgery. Six patients reported chronic pain, which disappeared after 0.5 year (4 patients), 3 years (1 patient), or 5 years (1 patient).

One patient reported an impairment of functional activities due to pain. This patient's neuropathic leg pain started soon after the procedure (age at surgery, 53 years) and became progressive over the final 10 years. This patient went to an outpatient pain clinic, but the treatment did not resolve the pain.

\section{Discontinuous chronic pain}

Discontinuous pain was reported by 13 patients (5\%). At this writing, 12 patients still experience discontinuous pain after a 10-year follow-up period. One of these patients with a recurrent inguinal hernia (patient 15, Fig. 2) was consulted during our physical examination at the outpatient clinic. Ultrasound examination was conclusive, and the patient underwent an open anterior mesh repair, after which the pain disappeared. One patient experienced pain, reportedly after much exercise, that started 5 years previously.

Figure 2 shows the chronic groin pain scores (VAS) for all the patients $(n=30)$ who had a period of continuous and discontinuous pain. The discontinuous pain had no influence on their daily activities.

Prognostic factors

Chronic groin pain was associated with preoperative pain $(P=0.03)$ but not with age, sex, type of hernia, bilateral hernia repair; primary or recurrent hernia, operation time, or hospital stay (Table 1). During long-term follow-up evaluation, seven recurrences were found (3\%). Chronic pain was not associated with recurrence $(P=0.4)$.

\section{Discussion}

The prevalence of chronic pain after endoscopic TEP hernia repair varies in the literature from 9 to $23 \%$ during a mean follow-up period ranging from 12 to 65 months [9, 18, 19]. In our study, a lower rate of chronic pain was found after a median follow-up period of 120 months. In the total study group, 17 patients $(6 \%)$ reported chronic groin pain, whereas only 10 patients (4\%) experienced pain after long-term follow-up evaluation. A total of 12 patients had some kind of complaint (discontinuous pain) after surgery that still was present after 10 years. Two patients had chronic continuous or discontinuous pain as a result of a recurrent inguinal hernia. For only one patient did continuous or discontinuous pain impair daily activities.

The population in the region where this study was performed has a high ratio of immigrants. This is the main reason why $14 \%$ of the patients were lost to follow-up evaluation.

The patients with preoperative pain reported significant more chronic pain $(P=0.03)$. This finding accords with the results of other studies $[8,13]$. The relation between preexisting pain and chronic pain may be explained by the fact that groin pain is not always treated by hernia repair and may not be caused by the operation or mesh
Fig. 2 Chronic pain score and period of continuous or discontinuous pain
- VAS, visual analogue score
Period of discontinuous pain (years)

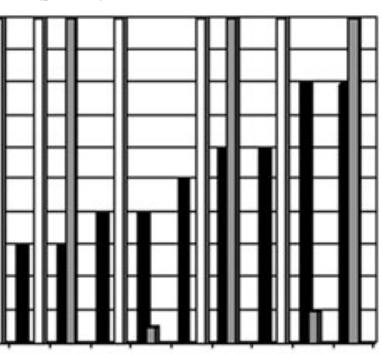


Table 1 Comparison of demographic features and perioperative data between the two groups of patients

a Seroma $(7 \%)$, scrotal hematoma (44\%), urinary retention (4\%), wound infection $(2 \%)$, nonstop bleeding $(9 \%)$, epididymitis $(2 \%)$, and hematoma of the groin $(33 \%)$

\begin{tabular}{|c|c|c|c|}
\hline & $\begin{array}{l}\text { Chronic pain group } \\
(n=17) n \text { (range) }\end{array}$ & $\begin{array}{l}\text { Pain-free group } \\
(n=256) n \text { (range) }\end{array}$ & $P$-value \\
\hline Median age (years) & $50(30-72)$ & $55(17-82)$ & 0.172 \\
\hline$>50$ & 8 & 158 & 0.346 \\
\hline Male/female & $16 / 1$ & $240 / 16$ & 1.0 \\
\hline Bilateral hernia & 1 & 39 & 0.482 \\
\hline Indirect/direct hernia & $8 / 6$ & $127 / 80$ & 0.976 \\
\hline Primary/recurrent hernia & $14 / 3$ & $226 / 30$ & 0.442 \\
\hline Patients with preoperative pain & 15 & 116 & 0.029 \\
\hline Operation performed by surgeon & 13 & 197 & 1.0 \\
\hline Median operation time & $38(15-90)$ & $35(14-108)$ & 0.569 \\
\hline Median hospital stay & $1(1-3)$ & $2(1-7)$ & 0.069 \\
\hline Complications $^{\mathrm{a}}$ & 3 & 57 & 1.0 \\
\hline Recurrence & 1 & 6 & 0.366 \\
\hline
\end{tabular}

replacement. However, detailed information on the history of preoperative pain, type of pain, and complaints involving other parts of the body is missing in the literature and in our study. The bias of our results could have been introduced by the telephone survey, although a verbal numeric rating score for pain should provide more reliable information than questionnaires [20].

It has been argued that the prosthetic mesh may contribute to the development of chronic groin pain [21]. In our previous study comparing mesh and nonmesh techniques, none of the patients from either group experienced persistent pain interfering with daily activity in the long term [2].

The results of this study show that for $35 \%$ (6/17) of the patients, chronic pain seemed to dissipate over time after endoscopic TEP hernia repair. Discontinuous pain (during several movements or after repetitive exercises) may be unpleasant but was not presented as a sufficient complaint by the patients. In our opinion, the only problematic case involved the patient with neuropathic leg pain and impairments due to his complaints.

\section{Conclusion}

The 10 years of follow-up evaluation seem to show that chronic groin pain after TEP repair of primary and recurrent inguinal hernia has a low incidence and intensity. The presentation and cause of groin pain after TEP is variable and probably not always caused by the procedure. Randomized controlled trials with long-term follow-up evaluation that includes and describes prospective, objective, qualitative pain measurements are needed.

Disclosures A. E. M. van der Pool, J. J. Harlaar, P. T. den Hoed, W. F. Weidema, and R.N. van Veen have no conflicts of interest or financial ties to disclose.
Open Access This article is distributed under the terms of the Creative Commons Attribution Noncommercial License which permits any noncommercial use, distribution, and reproduction in any medium, provided the original author(s) and source are credited.

\section{References}

1. Staarink M, van Veen RN, Hop WC, Weidema WF (2008) A 10year follow-up study on endoscopic total extraperitoneal repair of primary and recurrent inguinal hernia. Surg Endosc 22:1803-1806

2. van Veen RN, Wijsmuller AR, Vrijland WW, Hop WC, Lange JF, Jeekel J (2007) Long-term follow-up of a randomized clinical trial of nonmesh versus mesh repair of primary inguinal hernia. Br J Surg 94:506-510

3. Eklund AS, Montgomery AK, Rasmussen IC, Sandbue RP, Bergkvist LA, Rudberg CR (2009) Low recurrence rate after laparoscopic (TEP) and open (Lichtenstein) inguinal hernia repair: a randomized, multicenter trial with 5-year follow-up. Ann Surg 249:33-38

4. McCormack K, Scott NW, Go PM, Ross S, Grant AM (2003) Laparoscopic techniques versus open techniques for inguinal hernia repair. Cochrane Database Syst Rev (1):CD001785

5. Neumayer L, Giobbie-Hurder A, Jonasson O, Fitzgibbons R Jr, Dunlop D, Gibbs J, Reda D, Henderson W (2004) Open mesh versus laparoscopic mesh repair of inguinal hernia. N Engl J Med 350:1819-1827

6. Kehlet H, Bay-Nielsen M, Kingsnorth A (2002) Chronic postherniorrhaphy pain: a call for uniform assessment. Hernia 6:178181

7. Page B, Paterson C, Young D, O’Dwyer PJ (2002) Pain from primary inguinal hernia and the effect of repair on pain. Br J Surg 89:1315-1318

8. Poobalan AS, Bruce J, King PM, Chambers WA, Krukowski ZH, Smith WC (2001) Chronic pain and quality of life following open inguinal hernia repair. Br J Surg 88:1122-1126

9. Taylor CJ, Wilson T (2005) Long-term results of laparoscopic totally extraperitoneal inguinal herniorrhaphy. ANZ J Surg 75:637-639

10. Kehlet H, Jensen TS, Woolf CJ (2006) Persistent postsurgical pain: risk factors and prevention. Lancet 367:1618-1625

11. Wijsmuller AR, van Veen RN, Bosch JL, Lange JF, Kleinrensink GJ, Jeekel J (2007) Nerve management during open hernia repair. Br J Surg 94:17-22 
12. van Veen RN, Wijsmuller AR, Vrijland WW, Hop WC, Lange JF, Jeekel J (2007) Randomized clinical trial of mesh versus nonmesh primary inguinal hernia repair: long-term chronic pain at 10 years. Surgery 142:695-698

13. Wright D, Paterson C, Scott N, Hair A, O'Dwyer PJ (2002) Five-year follow-up of patients undergoing laparoscopic or open groin hernia repair: a randomized controlled trial. Ann Surg 235:333-337

14. Nienhuijs S, Staal E, Strobbe L, Rosman C, Groenewoud H, Bleichrodt R (2007) Chronic pain after mesh repair of inguinal hernia: a systematic review. Am J Surg 194:394-400

15. Liem MS, Halsema JA, van der Graaf Y, Schrijvers AJ, van Vroonhoven TJ (1997) Cost effectiveness of extraperitoneal laparoscopic inguinal hernia repair: a randomized comparison with conventional herniorrhaphy. Coala trial group. Ann Surg 226:668-675 (discussion 75-76)
16. Loos MJ, Roumen RM, Scheltinga MR (2007) Classifying postherniorrhaphy pain syndromes following elective inguinal hernia repair. World J Surg 31:1760-1765 (discussion 6-7)

17. Kehlet H (2008) Chronic pain after groin hernia repair. Br J Surg 95:135-136

18. Lau H, Patil NG, Yuen WK, Lee F (2003) Prevalence and severity of chronic groin pain after endoscopic totally extraperitoneal inguinal hernioplasty. Surg Endosc 17:1620-1623

19. Kumar S, Wilson RG, Nixon SJ, Macintyre IM (2002) Chronic pain after laparoscopic and open mesh repair of groin hernia. Br J Surg 89:1476-1479

20. Loos MJ, Houterman S, Scheltinga MR, Roumen RM (2008) Evaluating postherniorrhaphy groin pain: visual analogue or verbal rating scale? Hernia 12:147-151

21. Amid PK (2005) Groin hernia repair: open techniques. World J Surg 29:1046-1051 